\title{
Surface Analysis Due to Implantoplasty of Failed Dental Implants Using Laser Topography - An Experimental In-Vitro Study
}

\author{
Sahana Selvaganesh ${ }^{1}$, Subhasree R. ${ }^{2}$, Thiyaneswar Nessapan ${ }^{3}$, Abhinav R.P. ${ }^{4}$ Dimple S. ${ }^{5}$ \\ 1, 2,3,4,5 Department of Implantology, Saveetha Dental College and Hospitals, Saveetha Institute of Medical \\ and Technical Sciences, Saveetha University, Chennai, Tamil Nadu, India.
}

\section{ABSTRACT}

\section{BACKGROUND}

Peri-implantitis is an inflammatory reaction surrounding the hard and soft tissues of the implant that is functional in the patient's mouth. If left untreated, this might lead to further bone loss, loosening of the implant and the ultimate failure of the implant. There are many treatment modalities that have been suggested to treat periimplantitis, but there are no set guidelines or protocols for the same. Implantoplasty seems to be a viable option for treating peri-implantitis, as it involves the smoothening of the macro geometry of the supracrestal exposed implant surface thus reducing the plaque accumulation and ultimately preventing the bacterial recolonization and preventing the implant from failure. This study was done to evaluate the smoothness and the fracture resistance of the implants treated by implantoplasty and as an effective treatment for peri-implantitis.

\section{METHODS}

This in-vitro study was done on 5 failed implants, in which 4 were subjected to a sequence of dental burs and prophy paste and $1 \mathrm{implant}$ was kept as a control. They were subjected to laser topography analysis to determine the smoothness after implantoplasty, and their fracture resistance was also checked in Instron.

\section{RESULTS}

Implant no. 4 subjected to both dental burs and prophy paste was considered to be the smoothest with the laser topography of $2.049 \mathrm{~mm}$ compared to the control that was $3.132 \mathrm{~mm}$. Also, the fracture resistance between the implants and the control was similar.

\section{CONCLUSIONS}

In conclusion implantoplasty is a suggested treatment option for patients with periimplantitis keeping in mind the practical difficulties of the procedure.

\section{KEY WORDS}

Peri-Implantitis, Implantoplasty, Dental Burs, Surface Analysis
Corresponding Author:

Dr. Subhashree R.

Senior Lecturer,

Department of Implantology,

Saveetha Dental College and Hospitals,

Saveetha Institute of Medical and

Technical Sciences, Saveetha University,

Chennai, Tamil Nadu, India.

E-mail:drsubashree27@gmail.com

DOI: $10.14260 / j e m d s / 2021 / 471$

How to Cite This Article:

Selvaganesh S, Subhasree R, Nessapan T, et al. Surface analysis due to implantoplasty of failed dental implants using laser topography - an experimental in-vitro study. J Evolution Med Dent Sci 2021;10(30):2305-2309, DOI: $10.14260 / \mathrm{jemds} / 2021 / 471$

Submission 16-01-2021,

Peer Review 21-05-2021,

Acceptance 27-05-2021,

Published 26-07-2021.

Copyright (c) 2021 Sahana Selvaganesh et al. This is an open access article distributed under Creative Commons Attribution License [Attribution 4.0 International (CC BY 4.0)] 


\section{BACKGROUND}

Peri-implantitis is a condition where there is an inflammation that affects the hard and soft tissues surrounding the dental implant. This inflammation around the implants leads to pocket formation. ${ }^{1}$ Implantoplasty has been described by various authors, some proposed to polish the surface of the exposed dental implant which in turn would decontaminate the exposed surface and also reduce future bacterial colonization. ${ }^{2}$ The success of the dental implants and survival of the same has been on the increase but there are certain risk factors that would ultimately lead to the failure of the osseointegrated dental implants. ${ }^{3}$ Main factors that lead to failure of the osseointegrated implants is perimucositis followed by peri-implantitis.

Peri-implantitis causes progressive bone loss surrounding the implant followed by tissue loss which ultimately leads to aesthetic loss for the patient if in the anterior region of the oral cavity and implant failure is left untreated. Several treatments for implant decontamination are available. The treatments include detoxification of the exposed implant surface, ${ }^{4}$ debridement using non-surgical mechanical methods, ${ }^{5}$ debridement using surgical modifications, ${ }^{6}$ disinfection with chemotherapeutic agents, implant surface smoothening (implantoplasty) (air powder abrasion), ${ }^{7}$ laser therapy ${ }^{8}$ along with regenerative periodontal therapy can be performed accordingly to the type and extent of the peri-implantitis in that particular case. Carbon, plastic or titanium curettes, ultrasonic scaling or powder air abrasion all these methods can be used as mechanical methods for the detoxification of the implant surface. ${ }^{9}$

Implantoplasty is usually performed to flatten / smoothen the implant surface using rotary instruments such as dental intra oral burs, when an implant surface is exposed into the oral cavity and there is contamination with bacteria and plaque. ${ }^{7}$ This method of implantoplasty was recommended by Lang et al. and was reported by Suh et al. This recommended technique reduces the roughness of the titanium surface. Also there is decrease in the plaque adherence as it has been demonstrated and proved that rough surfaces tend to accumulate more plaque and bacteria in comparison with the smooth surface. Implant decontamination is the key solution for peri-implantitis treatment. Various mechanical and chemical methods have been tested and assessed in this respect. Implantoplasty is the method that has been introduced as one of the best methods to reduce plaque accumulation and inflammation which is associated with the exposed part of the titanium dental implant. This technique is done by rounding the threads and smoothening the part of the dental implant that is exposed in the oral cavity and with the use of dental rotary instruments. Diamonds, tungsten carbide, silicone polishers, and Arkansas stones have all been previously used for this procedure, they can be used either alone or in combination, to make the threads of the implant flat and smooth. In this technique, the surface roughness is changed, ${ }^{10}$ The surface of the implant that is important for adhesion of bone to the implant surface, macrostructure and the microstructure is removed. The resulting surface will depend upon the coarseness of the burs and the rotary instruments and also on the clinical skill of the dentist.
There are different methods to evaluate the surface smoothness after the implantoplasty procedure. Radiographic analysis pre and post-operative helps to evaluate the smoothness of the implant more effectively than the naked eye. Laser topography analysis is a method used to evaluate the pre- and post-operative surfaces. The process of implantoplasty comes with the fact that removing the threads of the exposed implants would actually weaken the strength of the implant in question. Thus, the fracture resistance of the implant was checked with the Instron machine. ${ }^{11}$ Here the object that has to be tested for fracture resistance is subjected to a load. This study was done to evaluate the smoothness and the fracture resistance of the implants treated by implantoplasty and as an effective treatment for periimplantitis.

\section{METHODS}

This in-vitro study was conducted in Saveetha Dental College and Hospitals after getting approval from the institutional review board. The implants were procured from the patients whose implants had failed. They were procured over a period of 1 year (Jan 2019 - Jan 2020). In this study, 5 failed implants were taken as the samples and they were randomly allocated under 4 different bur sequences, 1 implant was kept as the control and they were subjected to sterilisation, later these implants were mounted / inserted into the styrofoam model leaving 4 - 5 threads exposed (Fig. 1). A series of dental burs were used in the following sequence of black, green, blue, yellow followed by polishing with a prophy paste and polishing buffs. The burs which were selected for this were graded according to the coarseness. Pre-operative radiographs (Fig. 2) and laser topography analysis for each dental implant were done.
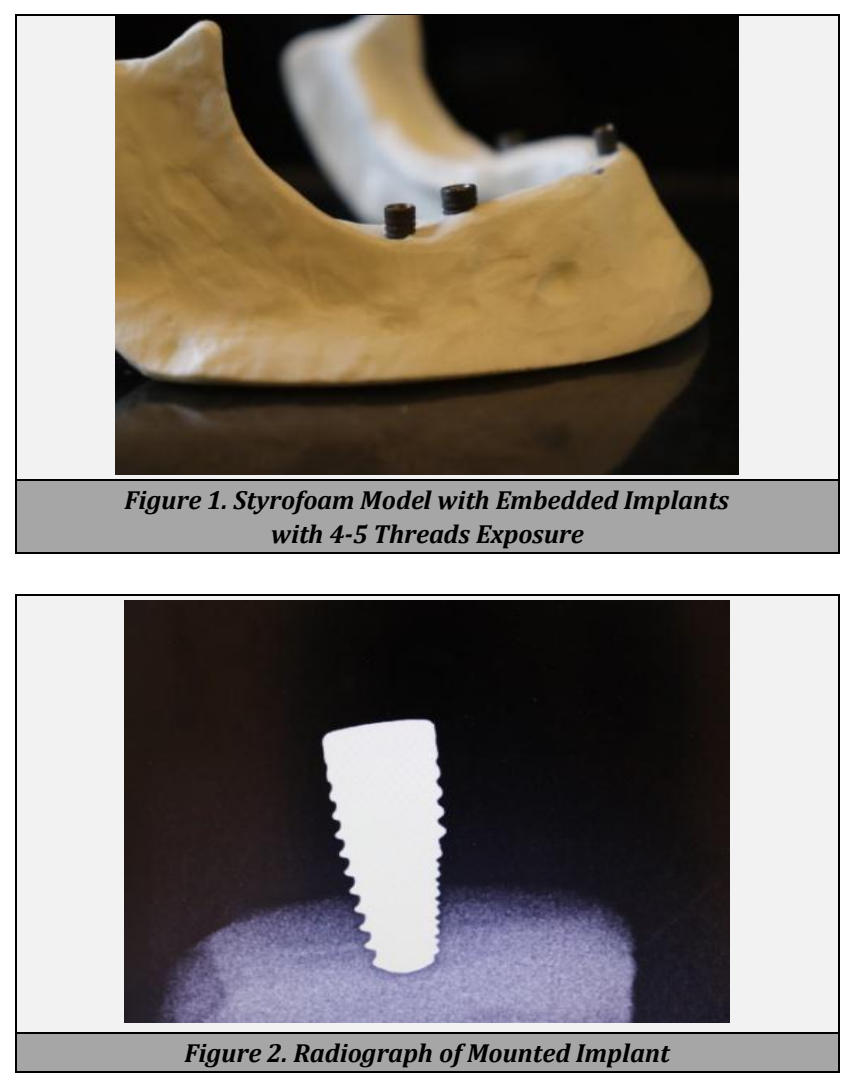
The sequence in which the burs were used by the operation on the mounted implants are as follows:

- IMPLANT 1: black and green band burs used.

- IMPLANT 2: black, green, blue band burs used.

- $\quad$ IMPLANT 3: black, green, blue and yellow band burs used.

- IMPLANT 4: All the burs plus polishing with prophy paste was done.

\section{- IMPLANT 5: Control}

During the procedure, the operator assessed certain factors such as 1 . Amount of titanium dust accumulated on the implant surface, 2. Ease of handling of the instruments and 3. Amount of smoothness according to the operator's eyes. Later the implantoplasty treated implants were mounted on to the putty index (Fig. 3) and were subjected to laser scanner analysis and the results were noted.

Stock abutments were screwed on to the implants and zirconia crowns were fabricated and the implants were subjected to fracture resistance test under Instron (Fig 4). The strength of the implant abutment complex ${ }^{12}$ was tested as implantoplasty involved removing the active threads, and care was taken to prevent the over reduction which could cause damage to the circumference of the implant and also the internal connections in the implant.
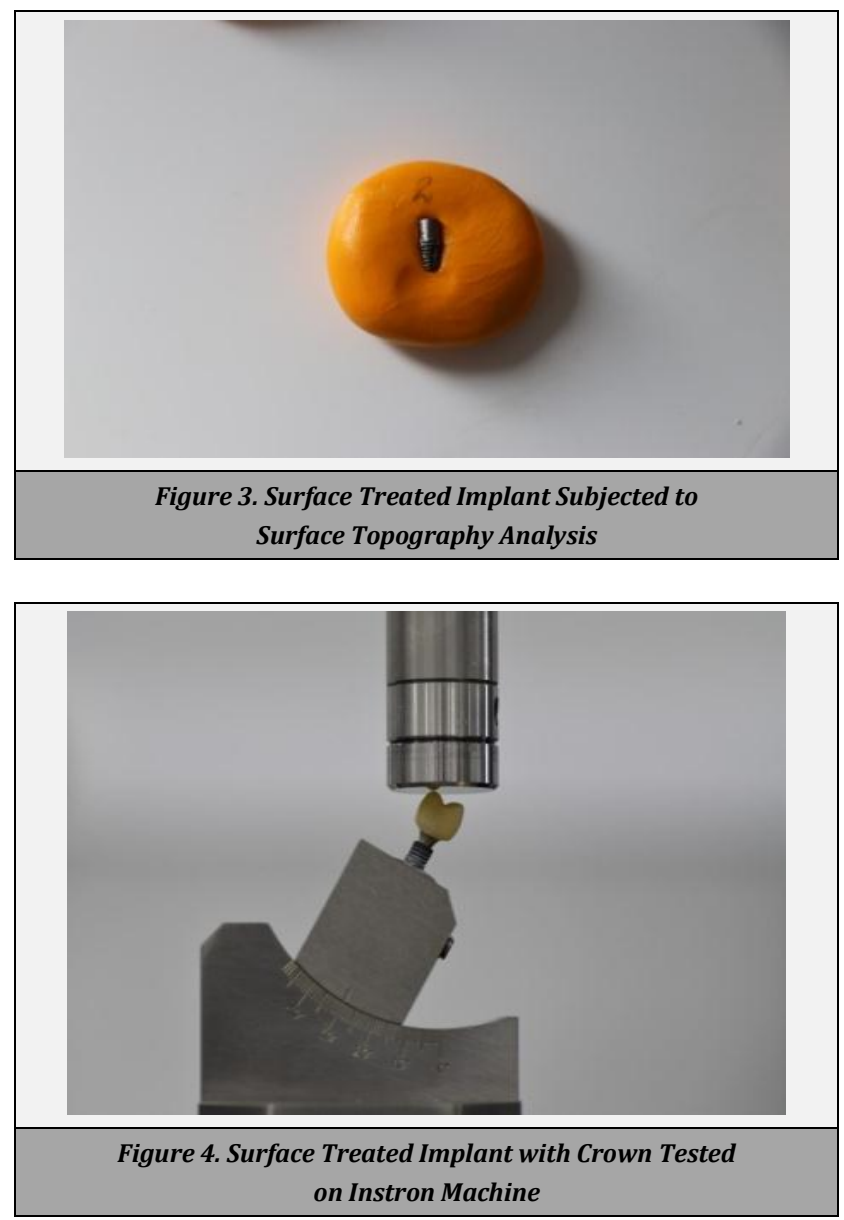

\section{Statistical Analysis}

The independent variables in this study were the burs used, operator skill, laser scanner and Instron. The dependent variables were the surface modification of dental implants.
After the results were obtained, statistical analysis was done, paired T test was done in SPSS software version 23.

\section{RESULTS}

Operator characteristics of amount of dust accumulated on the implant surface were evaluated according to the following - (0 - No amount of dust accumulated, 1 - Minimal amount of dust and slurry accumulated, 2 - Moderate amount of dust and slurry accumulated, 3 - Maximum amount of dust and slurry accumulated.)

- Implant - 1: 1

- Implant - 2: 2

- Implant - 3: 2

- Implant - 4:3

Amount of Titanium dust or slurry accumulated.

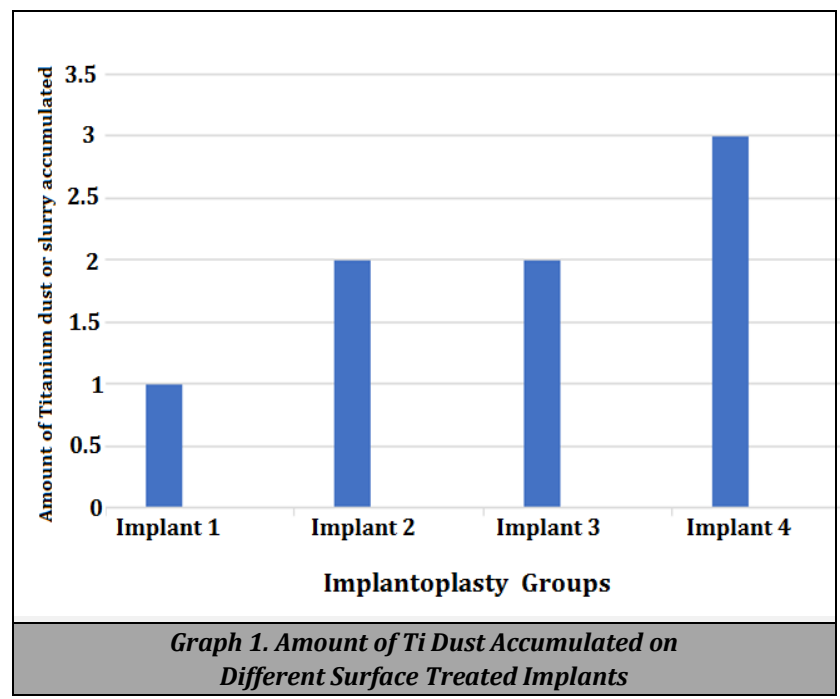

Smoothness of the implant on the naked eye was categorized as follows (1 - surface with harsh marks of the burs, 2 - moderately smooth surface, 3 - completely smooth surface to the naked eye)

- Implant 1:1

- Implant 2: 1

- Implant 3:2

- Implant 4: 3

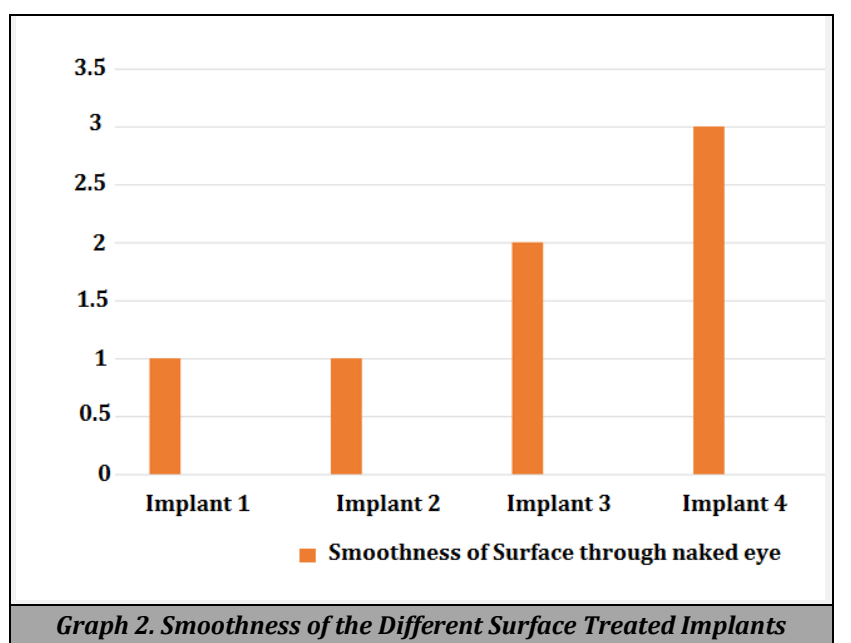


Laser scanner analysis was done and the surface of each of the implant was assessed.

\begin{tabular}{|cc|}
\hline Implantoplasty & $\begin{array}{c}\text { Surface Scanner Measurement } \\
(\mathbf{m m})\end{array}$ \\
Implant 1 & 3.056 \\
Implant 2 & 3.044 \\
Implant 3 & 3.043 \\
Implant 4 & 2.049 \\
Implant 5 & 3.132 \\
\hline Table 1. Surface Scanner Measurements for Surface Treated Implants \\
\hline
\end{tabular}

\section{DISCUSSION}

The progression of failure of implants start ${ }^{13}$ with the crestal bone loss then perimucositis ${ }^{14}$ which might lead to periimplantitis which occurs due to the accumulation of the biofilm on the rough surface of the exposed implant. ${ }^{15}$ There are several treatment options which are available for the treatment of peri-implantitis. The threads that are exposed in the oral environment should be rendered bacteria free, so that the plaque accumulation on the exposed implant surface has to be eliminated. One such procedure to render the site smooth is implantoplasty.

Implantoplasty's main aim is to provide a smooth surface for the implants so that the plaque does not adhere ${ }^{16,17}$ to the implant and the implants become devoid of threads and the surface becomes smoother. Previous in-vitro studies using diamond polishing devices have shown to remove the coating of the implant surface entirely in turn exposing the body of the fixture. ${ }^{10}$ In-vivo studies using diamond grit, carborundum polishing gives similar polished surfaces.

Various treatment options for treating peri-implantitis have been proposed in the literature with the primary aim to control the infection, smoothen the implant surface and regenerate alveolar bone. Ramel et al. suggested that the use of diamond burs have been proved to consume less time than that of the other types of implantoplasty techniques. Ramel et al. ${ }^{18}$ A plaque free implant surface is essential to achieve a positive treatment outcome. Implantoplasty has the ability to modify the macroscopic topography ${ }^{2}$ of the titanium implants to reduce retention of plaque and debris. No clear guidelines of armamentarium or clinical steps are available and is yet to be suggested.

There is no structured set of protocol for the bur selection for implantoplasty procedure. This in-vitro study classified different sequences of burs to be used and also added the use of prophy paste to get a more polished surface. There are several operator confounding factors that need to be taken into account and the results will vary accordingly. The speed at which the burs operate, the irrigation protocol also plays an important factor as there will be less heat that would be transferred to the implant and also copious irrigation will help to push out the titanium slurry and debris that gets collected.

The skill of the operator also plays an important role and the accuracy of handling the dental implants such that slurry or debris is not pushed deeper into the tissue and also the surrounding soft tissue and underlying bone is not disturbed.

The presence of residual debris may hamper the long-term health of the soft tissue surrounding the dental implant. This study has observed residual debris accumulation on the treated implant surface and thus final surface cleaning is required. Use of low abrasive air polishing powders such as glycine or erythritol or irrigation with saline may be considered good options which do not modify the implant surfaces further. ${ }^{19}$

In this study, along with polishing the implants irrigation was also used. While irrigation was being used the overheating of the implant during implantoplasty appeared to be minimized. A study has shown that only a minor change of $1.5^{\circ} \mathrm{C}$ in temperature occurs while irrigation is used. ${ }^{20}$ The debris remaining on the dental implant can be removed with the help of irrigation.

According to the study, the implant 4 turned out to be the smoothest and it was the implant which underwent implantoplasty with burs as well as the prophy paste. The surface scanner analysis with laser also suggested the same. The laser scanner that was used was SD Mechatronik. The vertical sensor resolution is up to $0,8 \mu \mathrm{m}$. Thus, fine structures such as cracks and traces of movement can be observed and resolved.

Use of prophy paste has enhanced the effectiveness of implantoplasty as a treatment of peri-implantitis. Prophy paste as a means of polishing after implantoplasty with dental burs has not been done. Prophy paste is usually used after scaling procedure to smoothen the tooth surface. The use of prophy paste with that of the polishing buffs rendered the surface even smoother than the others which used only burs.

\section{CONCLUSIONS}

This in-vitro study included 4 failed dental implants, which were sampled into 4 different implantoplasty sequence groups. In conclusion implantoplasty renders a smooth surface of the implants and the sequence of coarse to smooth burs along with prophy paste polishing was more effective than the other sequences. The fracture resistance of the dental implants which underwent implantoplasty was found to be similar to that of the implant that was kept as a control. This suggested that implantoplasty does not weaken the implant's strength. Implantoplasty can be an effective means for treating the implants with peri-implantitis, provided the operator's skill is efficient in preparing the implants without damaging the adjacent soft tissues and also the titanium debris and slurry and thoroughly irrigated out.

\section{Limitations}

The limitation of this study is the fact that implantoplasty was performed manually. When performed manually there is a lack of control over variables such as pressure and the times the rotating bur is in contact with the implant surface. In light of these conflicting results, future studies should aim in reducing these shortcomings and also assess the recolonization of the bacteria on the implantoplasty treated surface.

Data sharing statement provided by the authors is available with the full text of this article at jemds.com.

Financial or other competing interests: None.

Disclosure forms provided by the authors are available with the full text of this article at jemds.com. 


\section{REFERENCES}

[1] Morandi P, Azzola F, Cavalli N, et al. Implantoplasty in the surgical treatment of peri-implantitis: a case series. EAO Sepes

2017. http://dx.doi.org/10.26226/morressier.594928c4d462b 80296c9eed9

[2] Gustavo BL. Surface analisys of implants submitted to different methods of implantoplasty. http://dx.doi.org/10.26226/morressier.59492152d462 b80296c9f0df

[3] Shamsuddeen S, Kurien V. Failure in implants. Journal of Indian Dental Association 2020;14(3):31-8. http://dx.doi.org/10.33882/jida.14.25622

[4] Suarez F, Monje A, Galindo-Moreno P, et al. Implant surface detoxification. Implant Dent 2013;22(5):465-73.

[5] De Souza Junior JM, De Souza JGO, Neto ALP, et al. Analysis of effectiveness of different rotational instruments in implantoplasty. Implant Dent 2016;25(3):341-7.

[6] Romeo E, Lops D, Chiapasco M, et al. Therapy of periimplantitis with resective surgery. A 3-year clinical trial on rough screw-shaped oral implants. Part II: radiographic outcome. Clin Oral Implants Res 2007;18(2):179-87.

[7] Pighi J, Lombardo G, Marincola M, et al. Surgical regenerative treatments for peri-implantitis using a a desiccant with air powder abrasion followed by biphasic calcium sulfate grafting: a case series presentation. Journal of Clinical Periodontology 2018;45(S19):518-9. http://dx.doi.org/10.26226/morressier.5ac383282afee b00097a3df9

[8] Case Medical Research. Peri-implantitis, comparing treatments $970 \mathrm{~nm}$ laser and mucosal flap surgery. Case Medical Research 2020. http://dx.doi.org/10.31525/ct1 nct04249024

[9] Sahm N, Becker J, Santel T, et al. Non-surgical treatment of peri-implantitis using an air-abrasive device or mechanical debridement and local application of chlorhexidine: a prospective, randomized, controlled clinical study. J Clin Periodontol 2011;38(9):872-8.

[10] Tawse-Smith A, Kota A, Jayaweera Y, et al. The effect of standardised implantoplasty protocol on titanium surface roughness: an in-vitro study. Braz Oral Res 2016;30(1):e137.

[11] Gooch JW. Instron tensile tester. Encyclopedic dictionary of polymers. Springer 2011: p. 391.

[12] Chan HL, Oh WS, Ong HS, et al. Impact of Implantoplasty on strength of the implant-abutment complex. Int J Oral Maxillofac Implants 2013;28(6):1530-5.

[13] Heitz-Mayfield L, Needleman I, Salvi G, et al. Consensus statements and clinical recommendations for prevention and management of biologic and technical implant complications. Int J Oral Maxillofacial Implants 2014;29 Suppl:346-50.

[14] Swierkot K, Lottholz P, Flores-de-Jacoby L, et al. Mucositis, peri-implantitis, implant success and survival of implants in patients with treated generalized aggressive periodontitis: 3-to 16-year results of a prospective longterm cohort study. J Periodontol 2012;83(10):1213-25.

[15] Burgers R, Gerlach T, Hahnel S, et al. In vivoandin vitrobiofilm formation on two different titanium implant surfaces. Clin Oral Implants Res 2010;21(2):156-64.

[16] Demling A, Heuer W, Elter C, et al. Analysis of supra-and subgingival long-term biofilm formation on orthodontic bands. Eur J Orthod 2009;31(2):202-6.

[17] Renvert S, Polyzois I, Claffey N. How do implant surface characteristics influence peri-implant disease? J Clin Periodontol 2011;38(Suppl 11):214-22.

[18] Ramel CF, Lüssi A, Özcan M, et al. Surface roughness of dental implants and treatment time using six different implantoplasty procedures. Clin Oral Implants Res 2016;27(7):776-81.

[19] Schmidt KE, Auschill TM, Heumann C, et al. Influence of different instrumentation modalities on the surface characteristics and biofilm formation on dental implant neck, in vitro. Clin Oral Implants Res 2017;28(4):483-90.

[20] Sharon E, Shapira L, Wilensky A, et al. Efficiency and thermal changes during implantoplasty in relation to bur type. Clin Implant Dent Relat Res 2013;15(2):292-6. 